\title{
ULTRAPOTENTIALS AND POSITIVE EIGENFUNCTIONS FOR AN ABSOLUTELY CONTINUOUS RESOLVENT OF KERNELS
}

\author{
LUCIAN BEZNEA
}

\section{Intro duction}

I et $(X, \mathscr{B})$ be a measurable space and $\mathscr{V}$ be a submarkovian resolvent of kernels (with the initial kernel $V$ proper) on $X$ which is absolutely continuous and has a dual resolvent (with the same properties) with respect to a $\sigma$-finite measure.

A positive numerical function $s$ on $X$ is called $V$-ultrapotential if it is $\mathscr{V}$-excessive (in particular $\mathscr{V}$-a.e. finite) and if the following condition is fulfilled: for every integer $n \geq 1$, there exists a positive $\mathscr{B}$-measurable function $f_{n}$ on $X$ such that $s=V^{n}\left(f_{n}\right)$, where $V^{n}$ is the $n$-th iteration of the kernel $V$.

The main purpose of this paper (see Theorem 3.5 and Corollary 3.6) is to prove that, under a "regularity" condition (which will be discussed in the last part of Section 2) on the resolvent $\mathscr{V}$, for each $V$-ultrapotential $s$ there exist a finite positive Borel measure $\sigma$ on the open interval ]0, $\infty$ [ and a family $\left(s_{\lambda}\right)_{0<\lambda<\infty}, s_{\lambda}$ being a positive $\lambda$-eigenfunction of $V$ (i.e. $V\left(s_{\lambda}\right)=\lambda \cdot s_{\lambda}$ and $s_{\lambda}$ is $\mathscr{V}$-a.e. finite), for any $\lambda>0$, such that for each $x \in X$ the numerical function $\lambda \mapsto s_{\lambda}(x)$, defined on $] 0, \infty[$, is $\sigma$-measurable and

$$
s(x)=\int s_{\lambda}(x) d \sigma(\lambda)
$$

In fact, this type of representation is given for a slightly more general class of excessive functions, as the $V$-ultrapotentials.

An uniqueness of the representation and a converse statement are also proved.

These results ar: analogous, in this context, with those obtained by M. Itô and N. Suzuki in [7] (see also [6]) for the set up of diffusion semi-

Recei red May 6, 1987. 
groups. (The $V$-ultrapotentials are corresponding to the completely $A$ superharmonic measures with zero conditions considered in [7]).

We shall use the technique developed in [6] and [7] and also the duality theory for standard $H$-cones (see [1]).

As in [7], the Bernstein theorem on complete monotone functions is a consequence of these results (see $\S 4$, Example 1). Another one is that the convex cone of $V$-ultrapotentials is one dimensional if the resolvent satisfies an ellipticity condition (Example 2 in $\S 4$ ).

Let us notice that the existence of the dual resolvent is not essential for our aim. This assumption may be substituted by the duality theory of standard $H$-cones (see Remark 3.9).

\section{$\S 1$. Ultrapotentials-first results}

In this section, $\mathscr{V}=\left(V_{\alpha}\right)_{\alpha>0}$ will be a submarkovian resolvent of kernels (on the measurable space $(X, \mathscr{B})$ ) with the initial kernel $V$ proper.

We denote by $\mathscr{E}_{r}$ the usual ordered convex cone of $\mathscr{V}$-a.e. finite excessive functions associated to $\mathscr{V}$. (For more details see [1] or [8]).

Definition. A $\mathscr{V}$-excessive function $s \in \mathscr{E}_{\curlyvee}$ is called $V$-ultrapotential iff: for any integer $n \geq 1$ there exists a positive $\mathscr{B}$-measurable (numerical) function $f_{n}$ on $X$ such that $s=V^{n}\left(f_{n}\right)$.

In the above definition, the function $f_{n}$ may be chosen to be $\mathscr{V}$ excessive and in this case it is uniquely defined and denoted by $s^{n}$.

Let us denote by $\mathscr{U}(V)$ the convex cone of all $V$-ultrapotentials. Thus:

$$
\begin{aligned}
& \mathscr{U}(V)=\left\{s \in \mathscr{E}_{r} / \text { for any integer } n \geq 1 \text { there exists } s^{n} \in \mathscr{E}_{\mathscr{V}}\right. \text { such that } \\
&\left.s=V^{n}\left(s^{n}\right)\right\} .
\end{aligned}
$$

Remark 1.1. $\mathscr{U}(V)$ generalises the convex cone of "ultrapotentials associated to a second order elliptic differential operator" from [2]. (See Corollary 4.c) in [2]).

For an excessive function $s \in \mathscr{E}_{\curlyvee}$ we have: $s \in \mathscr{U}(V)$ iff there exists (uniquely) a sequence $\left(s^{n}\right)_{n \geq 0} \subseteq \mathscr{E}_{\vee}$ such that $s^{n}=V\left(s^{n+1}\right)$, for any integer $n \geq 0$, where $s^{0}=s$.

If $\mathscr{E}$ is an ordered convex cone, we denote by $[\mathscr{E}]$ the ordered vector space canonically generated by $\mathscr{E}$ (see $\S 2.1$ in [1]). 
Definition. If $\alpha \geq 0$ and $\beta>0$ are real numbers, we define the map $\phi_{\beta}^{\alpha}$ from $\mathscr{E}_{\psi}$. into $\left[\mathscr{E}_{\gamma}\right]$ by:

$$
\phi_{\beta}^{\alpha}(s)=s-\beta V_{\alpha+\beta}(s), \quad s \in \mathscr{E}_{\gamma} .
$$

For every $\alpha \geq 0, \beta>0, \phi_{\beta}^{\alpha}$ and $V_{\beta}$ being additive and positive homogeneous, we may extend them to linear operators on $\left[\mathscr{E}_{\gamma}\right]$.

Definition. Let $\alpha \geq 0$ be fixed. We denote by $\tilde{\mathscr{U}}_{\alpha}(V)$ the totality of those excessive functions $s$ which for any integer $n \geq 1$ and $\beta_{1}, \cdots, \beta_{n}>0$ satisfy: $\left(\phi_{\beta_{1}}^{\alpha} \circ \cdots \circ \phi_{\beta_{n}}^{\alpha}\right)(s) \in \mathscr{E}_{r}$.

For any $\alpha \geq 0, \tilde{\mathscr{U}}_{\alpha}(V)$ is a convex subcone of $\mathscr{E}_{r}$. We write $\tilde{\mathscr{U}}(V)$ instead of $\tilde{\mathscr{U}}_{0}(V)$.

Paper [3] has already underlined the convex cone $\tilde{\mathscr{U}}(V)$ (denoted there by $\mathscr{S}_{\infty}$, its elements being called " $\mathscr{V}$-complete supermean functions") and has studied it under other points of view.

Remark 1.2. If $0 \leq \alpha_{1} \leq \alpha_{2}$ and $\beta>0$, then

$$
\begin{gathered}
\phi_{\beta}^{\alpha_{2}}(s)=\phi_{\beta}^{\alpha_{1}}(s)+\beta\left(\alpha_{2}-\alpha_{1}\right) V_{\alpha_{1}+\beta}\left(V_{\alpha_{2}+\beta}(s)\right), \quad s \in \mathscr{E}_{\gamma} ; \\
\phi_{\beta}^{\alpha_{2}}\left(\tilde{\mathscr{U}}_{\alpha_{1}}(V)\right) \subseteq \tilde{\mathscr{U}}_{\alpha_{1}}(V) ; \\
\tilde{\mathscr{U}}_{\alpha_{1}}(V) \subseteq \tilde{\mathscr{U}}_{\alpha_{2}}(V) .
\end{gathered}
$$

Proof. (1.2) is a consequence of (1.1) since for any $\alpha \geq 0$ and $\beta>0$ : $V_{\beta}\left(\tilde{\mathscr{U}}_{\alpha}(V)\right) \subseteq \tilde{\mathscr{U}}_{\alpha}(V), \phi_{\beta}^{\alpha}\left(\tilde{\mathscr{U}}_{\alpha}(V)\right) \subseteq \tilde{\mathscr{U}}_{\alpha}(V)$.

In order to establish other connections between the convex cones $\mathscr{U}(V)$ and $\tilde{\mathscr{U}}_{\alpha}(V), \alpha \geq 0$, let us recall from [9] that any $\mathscr{V}$-excessive function $s$ has an unique decomposition $s=s^{\prime}+s^{\prime \prime}$, where $s^{\prime}$ is an invariant function (i.e. $\alpha V_{\alpha}\left(s^{\prime}\right)=s^{\prime}, \alpha>0$ ) and $s^{\prime \prime}$ is purely excessive (i.e. $\bigwedge_{\alpha>0} \alpha V_{\alpha}\left(s^{\prime \prime}\right)=0$, the sign " $\wedge$ " having the usual meaning of lattice operation in $\left.\mathscr{E}_{r}\right)$.

Remark 1.3. a) Let $s \in \mathscr{E}_{\curlyvee}$ be such that one of the following two conditions is satisfied:

(i) $V(s) \in \mathscr{E}_{\gamma}$;

(ii) $s=V(f)$, where $f$ is a positive measurable numerical function. Then $s$ is purely excessive. In addition, the function $f$ is $\mathscr{V}$-a.e. finite.

b) Any $V$-ultrapotential is a purely excessive function.

Proposition 1.4. a) If $\alpha_{0} \geq 0$, then:

$$
\tilde{\mathscr{U}}_{\alpha_{0}}(V)=\bigcap_{\alpha>\alpha_{0}} \tilde{\mathscr{U}}_{\alpha}(V) \text {. }
$$


b) For every $\alpha>0$ we have:

$\tilde{\mathscr{U}}_{\alpha}(V)=\left\{s \in \mathscr{E}_{r} /\right.$ for any integer $n \geq 1$ there exists $s_{\alpha}^{n} \in \mathscr{E}_{r}$ such that $\left.s=V_{\alpha}^{n}\left(s_{\alpha}^{n}\right)\right\}$.

c) The V-ultrapotentials are exactly the purely excessive elements of $\tilde{\mathscr{U}}(V)$.

Proof. If $\alpha_{1}>\alpha_{0}$ and $\beta>0$, from (1.2) and (1.3) it follows that

$$
\phi_{\beta}^{\alpha_{1}}\left(\bigcap_{\alpha>\alpha_{0}} \tilde{\mathscr{U}}_{\alpha}(V)\right) \subseteq \bigcap_{\alpha>\alpha_{0}} \tilde{\mathscr{U}}_{\alpha}(V) .
$$

As a consequence, if $s \in \bigcap_{\alpha>\alpha_{0}} \tilde{\mathscr{U}}_{\alpha}(V)$ and $\beta>0$, using also (1.1), it follows successively: $\phi_{\beta}^{\alpha_{0}}(s)=\bigwedge_{\alpha>\alpha_{0}} \phi_{\beta}^{\alpha}(s) \in \mathscr{E}_{r}, \phi_{\beta}^{\alpha_{0}}(s) \in \bigcap_{\alpha>\alpha_{0}} \tilde{\mathscr{U}}_{\alpha}(V), s \in \tilde{\mathscr{U}}_{\alpha_{0}}(V)$. Thus, assertion a) is clear, using also (1.3).

We denote by $\mathscr{U}_{\alpha}(V)$ the set of the right side in the equality of $b$ ). First, we show that, if $\alpha \geq 0$ and $\beta>0$, then:

$$
\phi_{\beta}^{\alpha}\left(\mathscr{U}_{\alpha}(V)\right) \subseteq \mathscr{U}_{\alpha}(V),
$$

where $\mathscr{U}_{0}(V)=\mathscr{U}(V)$.

Indeed, let $n$ be an integer, $n \geq 1$ and $s \in \mathscr{U}_{\alpha}(V)$. Then:

$$
\begin{aligned}
\phi_{\beta}^{\alpha}(s) & =V_{\alpha}^{n}\left(V_{\alpha}\left(s_{\alpha}^{n+1}\right)\right)-\beta V_{\alpha+\beta}(s)=V_{\alpha}^{n}\left(V_{\alpha+\beta}\left(s_{\alpha}^{n+1}\right)+V_{\alpha}\left(\beta V_{\alpha+\beta}\left(s_{\alpha}^{n+1}\right)\right)\right)-\beta V_{\alpha+\beta}(s) \\
& =V_{\alpha}^{n}\left(V_{\alpha+\beta}\left(s_{\alpha}^{n+1}\right)\right)
\end{aligned}
$$

i.e. (1.4) is proved. As a consequence, for any $\alpha \geq 0$ : $\mathscr{U}_{\alpha}(V) \subseteq \tilde{\mathscr{U}}_{a}(V)$.

Let us now prove that: if $s \in \tilde{\mathscr{U}}_{\alpha}(V), \alpha>0$, (resp. $s \in \tilde{\mathscr{U}}(V)$ and $s$ is purely excessive) then $s \in \mathscr{U}_{\alpha}(V)$ resp. $\left.s \in \mathscr{U}(V)\right)$.

From $\phi_{\beta}^{\alpha}(s) \in \mathscr{E}_{r}$, for every $\beta>0$, it results:

$$
\beta \phi_{\beta}^{\alpha}(s) \leq \beta^{\prime} \phi_{\beta^{\prime}}^{\alpha}(s), \quad \text { for } \beta \leq \beta^{\prime}
$$

and we define the function $\bar{s}_{\alpha}$ by:

$$
\bar{s}_{\alpha}=\sup _{\beta>0} \beta \phi_{\beta}^{\alpha}(s) \text {. }
$$

If $s_{1}, s_{2} \in \tilde{\mathscr{U}}_{a}(V), \alpha \geq 0$, then:

$$
\overline{\left(s_{1}\right)_{\alpha}}+\overline{\left(s_{2}\right)_{\alpha}}=\overline{\left(s_{1}+s_{2}\right)_{\alpha}} .
$$

If $s \in \tilde{\mathscr{U}}_{\alpha}(V), \alpha>0$, (resp. $s \in \tilde{\mathscr{U}}(V)$ and $s$ is purely excessive) then: $V_{\alpha}\left(\bar{s}_{\alpha}\right)=\sup _{\beta>0} \beta V_{\alpha}\left(s-\beta V_{\alpha+\beta}(s)\right)=\sup _{\beta>0} \beta V_{\alpha+\beta}(s)=s$. By Remark 1.3 a) it follows that $\bar{s}_{\alpha} \in \mathscr{E}_{r}$ if $\alpha>0$ (resp. $\bar{s}_{0} \in \mathscr{E}_{r}$ and $s_{0}$ is purely excessive.). From $s=\phi_{\beta}^{\alpha}(s)+\beta V_{\alpha+\beta}(s), \phi_{\beta}^{\alpha}(s) \in \tilde{\mathscr{U}}_{\alpha}(V), \beta V_{\alpha+\beta}(s) \in \tilde{\mathscr{U}}_{\alpha}(V)$, using (1.5), it 


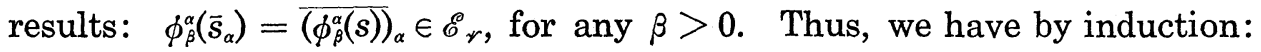
$\bar{s}_{\alpha} \in \tilde{\mathscr{U}}_{\alpha}(V), s \in \mathscr{U}_{\alpha}(V)$. Assertion b) is now clear.

Finally, assertion c) follows from the above considerations and Remark 1.3 b).

Definition. a) For every real numbers $\alpha \geq 0$ and $\lambda>0$, the convex cone $E_{\alpha}(V, \lambda)$ of all positive $\lambda$-eigenfunctions of $V_{\alpha}$ is defined by:

$$
E_{\alpha}(V, \lambda)=\left\{s \in \mathscr{E}_{r} / V_{\alpha}(s)=\lambda \cdot s\right\},
$$

where $V_{0}=V$, and we write $E(V, \lambda)$ instead of $E_{0}(V, \lambda)$.

b) Let us denote by $E(V, \infty)$ the convex cone of all invariant functions. Hence

$$
E(V, \infty)=E_{\alpha}\left(V, \frac{1}{\alpha}\right), \quad \text { for any } \alpha>0
$$

We remark that:

$$
E_{\alpha}(V, \lambda)=\{0\} \quad \text { if } \alpha>0 \text { and } \frac{1}{\alpha}<\lambda .
$$

From the resolvent equation, it is easy to see that for $\alpha, \beta \geq 0$ we have:

$$
E_{\alpha}(V, \lambda)=E_{\beta}\left(V, \frac{\lambda}{1+(\beta-\alpha) \lambda}\right) \quad \text { if } 0<\lambda \leq \frac{1}{\alpha}
$$

where the convention is: $\frac{1}{0}=\infty, \frac{\infty}{1+\beta \infty}=\frac{1}{\beta}, E_{0}(V, \infty)=E(V, \infty)$.

Let us point out that

$$
\bigcup_{0<\lambda \leq \infty} E(V, \lambda) \subseteq \tilde{\mathscr{U}}(V)
$$

and by (1.6) we have

$$
\bigcup_{0<\lambda \leq \infty} E(V, \lambda)=\bigcup_{0<\lambda \leq 1 / \alpha} E_{\alpha}(V, \lambda), \quad \text { for any } \alpha>0 .
$$

\section{§2. Resolvents in duality and the natural topology}

Let $\mathscr{V}=\left(V_{\alpha}\right)_{\alpha>0}$ and $\mathscr{W}=\left(W_{\alpha}\right)_{\alpha>0}$ be two submarkovian resolvents of kernels (on the measurable space $(X, \mathscr{B})$ ) which are absolutely continuous and in duality with respect to a $\sigma$-finite measure $m$, their initial kernels $V$ and $W$ being proper. 
At first we summarize generalities of standard $H$-cones. For more details and proofs one should consult [1].

$\mathscr{E}_{\gamma}$ and $\mathscr{E}_{\text {}}$ stand as basic examples of standard $H$-cones.

In order to simplify the exposition, let us suppose that $v=W(1)$ is a function on $X$ with strictly positive values. We write: $v>0$.

Definition. a) Let us fix $u \in \mathscr{E}_{\mathscr{W}}, u>0$. An element $t \in \mathscr{E}_{\mathscr{W}}$ is called $u$-continuous if for any $\varepsilon>0$ and for any family $F \subseteq \mathscr{E}_{\mathscr{r}}$ increasing to $t$ there exists $t_{1} \in F$ such that $t \leq t_{1}+\varepsilon \cdot u$.

The convex cone of all $u$-continuous elements of $\mathscr{E}_{\mathscr{W}}$ is denoted by $\left(\mathscr{E}_{\mathscr{r}}\right)_{0}(u)$.

b) An element $t \in \mathscr{E}_{r}$ is called universally continuous if it is $u$ continuous for every $u \in \mathscr{E}_{w}, u>0$.

We denote by $\left(\mathscr{E}_{\Downarrow}\right)_{0}$ the convex cone of all universally continuous elements of $\mathscr{E}_{\mathscr{w}}$.

(2.1) There exists a countable subset $D$ of $\left(\mathscr{E}_{r}\right)_{0}$ which is increasingly dense in $\mathscr{E}_{\mathscr{V}}$ (i.e. for every $t \in \mathscr{E}_{\mathscr{V}}$ there exists a sequence $\left(t_{n}\right)_{n \in N} \subset D$ such that $\left.t_{n} \nearrow t\right)([1]$, Theorem 4.4.6).

(2.2) For any $u \in \mathscr{E}_{\mathscr{w}}, u>0$ and $t \in\left(\mathscr{E}_{\mathscr{r}}\right)_{0}$, there exists $a \in \boldsymbol{R}_{+}$such that $t \leq a u$. ([1], Proposition 4.1.2. b))

Definition. The energy form is the map $(s, t) \mapsto\langle s, t\rangle$ from $\mathscr{E}_{\mathscr{r}} \times \mathscr{E}_{\mathscr{r}}$ into $\overline{\boldsymbol{R}}_{+}$defined by:

$$
\langle s, t\rangle=\sup \left\{\int f W(g) d \mathfrak{m} / f, g \in \mathscr{F}, V(f) \leq s, W(g) \leq t\right\},
$$

where $\mathscr{F}$ is the set of all $\mathscr{B}$-measurable positive numerical functions on X.

(2.3) For any $f \in \mathscr{F}$ such that $V(f) \in \mathscr{E}_{\gamma}$ and for any $t \in \mathscr{E}_{\mathscr{r}}$, we have:

$$
\langle V(f), t\rangle=\int f t d \mathfrak{m} \text {. }
$$

([1], Theorem 1.2.2 a))

Definition. An $H$-integral on $\mathscr{E}_{\mathscr{V}}$ is a map $\mu$ from $\mathscr{E}_{\mathscr{V}}$ into $\overline{\boldsymbol{R}}_{+}$with the following properties:

$\mu$ is additive;

$\mu$ is increasing;

$\mu$ is continuous in order from below (i.e. if $\left(t_{n}\right)_{n \in N} \subseteq \mathscr{E}_{\mathscr{W}}$ increases to $t \in \mathscr{E}_{\mathscr{W}}$, then $\left(\mu\left(t_{n}\right)\right)_{n \in N}$ increases to $\left.\mu(t)\right)$; 
there exists $u \in \mathscr{E}_{\mathscr{w}}, u>0$ such that $\mu(u)<\infty$ (or equivalent: $\mu(t)<\vdots_{\infty}$ for every $\left.t \in\left(\mathscr{E}_{\nVdash}\right)_{0}\right)$.

Definition. If $s \in \mathscr{E}_{r}$, the map $\tilde{s}$ from $\mathscr{E}_{\mathscr{V}}$ into $\overline{\boldsymbol{R}}_{+}$is defined by:

$$
\tilde{s}(t)=\langle s, t\rangle, \quad \text { for any } t \in \mathscr{E}_{\mathscr{r}} .
$$

For every $s \in \mathscr{E}_{r}, \tilde{s}$ is an $H$-integral on $\mathscr{E}_{r}$ ([1], Theorem 1.2.2).

For every $H$-integral $\mu$ on $\mathscr{E}_{\psi}$ there exists an uniquely defined $s \in \mathscr{E}_{\gamma}$ such that $\mu=\tilde{s}$. ([1], Proposition 1.2.3).

(2.4) Hence, by means of the energy form we may identify $\mathscr{E}_{r}$ (resp. $\mathscr{E}_{\Downarrow}$ ) with the set of all $H$-integrals on $\mathscr{E}_{r}$ (resp. on $\mathscr{E}_{r}$ ).

(2.5) An additive and increasing map $\mu:\left(\mathscr{E}_{r}\right)_{0} \rightarrow \boldsymbol{R}_{+}$is the restriction to $\left(\mathscr{E}_{\psi}\right)_{0}$ of an $H$-integral on $\mathscr{E}_{\mathscr{w}}$. ([1], Theorem 4.2.11)

The resolvents $\mathscr{V}=\left(V_{\alpha}\right)_{\alpha>0}$ and $\mathscr{W}=\left(W_{\alpha}\right)_{\alpha}$ being in duality, from Hunt's approximation theorem and (2.3) it follows:

$$
\left\langle V_{\alpha}(s), t\right\rangle=\left\langle s, W_{\alpha}(t)\right\rangle,
$$

for every $\alpha>0, s \in \mathscr{E}_{\nu}$ and $t \in \mathscr{E}_{\psi}$.

Definition. The natural topology on $\mathscr{E}_{\nu}$ is the coarsest topology which makes continuous the maps $s \mapsto\langle s, t\rangle, t \in\left(\mathscr{E}_{y}\right)_{0}$.

(2.7) The vector spaces $\left[\mathscr{E}_{r}\right]$ and $\left[\left(\mathscr{E}_{r}\right)_{0}\right]$ are in duality (by means of the energy form) and the natural topology on $\mathscr{E}_{r}$ is the restriction to $\mathscr{E}_{r}$ of the weak topology $\sigma\left(\left[\mathscr{E}_{r}\right],\left[\left(\mathscr{E}_{r}\right)_{0}\right]\right)$. ([1], page 106)

The natural topology on $\mathscr{E}_{r}$ is metrisable. ([1], Proposition 4.2.8)

Definition. For any $u \in \mathscr{E}_{\mathscr{w}}, u>0$ we put:

$$
K_{u}=\left\{s \in \mathscr{E}_{r} \mid\langle s, u\rangle \leq 1\right\} \text {. }
$$

If $u \in \mathscr{E}_{r}, u>0$, then $K_{u}$ is a compact convex subset of $\mathscr{E}_{r}$.

(2.8) The coarsest topology on $\mathscr{E}_{r}$ which makes continuous all the functions $s \mapsto\langle s, t\rangle, t \in\left(\mathscr{E}_{w}\right)_{0}(u)$ coincide with the natural topology on $K_{u}$. (See the proof of Proposition 4.2.4 in [1]). In addition

$$
\mathscr{E}_{r}=\bigcup_{\substack{u \in \mathbb{E}_{w} \\ u>0}} K_{u} .
$$

([1], Proposition 4.2.3 a))

(2.10) A subset $M$ of $\mathscr{E}_{r}$ is relatively compact in the natural topology iff there exists $u \in \mathscr{E}_{w}, u>0$ such that $M \subseteq K_{u}$ ([1], Proposition 4.2.7).

Let us now discuss some "regularity" properties of the resolvents. 
Definition. a) Let $u \in \mathscr{E}_{w}, u>0$ be fixed. We say that the resolvent $\mathscr{V}=\left(V_{\alpha}\right)_{\alpha>0}$ satisfies condition $\left(\mathscr{R}_{u}\right)$ if:

$V_{\alpha}: K_{u} \rightarrow \mathscr{E}_{\gamma}$ is continuous (in the natural topology), for any $\alpha>0$.

b) The resolvent $\mathscr{V}=\left(V_{\alpha}\right)_{\alpha>0}$ satisfies condition $(\mathscr{R})$ if:

$$
V_{\alpha}: \mathscr{E}_{\gamma} \rightarrow \mathscr{E}_{\gamma} \text { is continuous, for every } \alpha>0 \text {. }
$$

The dual properties are the following:

Definition. a) If $u \in \mathscr{E}_{\mathscr{W}}, u>0$, the resolvent $\mathscr{W}=\left(W_{\alpha}\right)_{\alpha>0}$ satisfies condition $\left(\mathscr{R}_{u}^{*}\right)$ if:

$$
W_{\alpha}\left(\left(\mathscr{E}_{\#}\right)_{0}\right) \subseteq\left(\mathscr{E}_{w}\right)_{0}(u), \quad \text { for any } \alpha>0 .
$$

b) The resolvent $\mathscr{W}=\left(W_{\alpha}\right)_{a>0}$ satisfies condition ( $\left.\mathscr{R}^{*}\right)$ if:

$$
W_{\alpha}\left(\left(\mathscr{E}_{\nVdash}\right)_{0}\right) \subseteq\left(\mathscr{E}_{\psi}\right)_{0}, \quad \text { for any } \alpha>0 \text {. }
$$

In [1], page 150, the maps $W_{\alpha}, \alpha>0$ are called regular if condition $(\mathscr{R} *)$ is satisfied.

From (2.10) we have: $\mathscr{V}$ satisfies condition $(\mathscr{R})$ (resp. $\mathscr{W}$ satisfies condition $\left(\mathscr{R}^{*}\right)$ ) iff $\mathscr{V}$ satisfies $\left(\mathscr{R}_{u}\right)$ (resp. $\mathscr{W}$ satisfies $\left(\mathscr{R}_{u}^{*}\right)$ ) for every $u \in \mathscr{E}_{w}, u>0$.

By (2.6) and (2.7) it is easy to see that:

$$
(\mathscr{R}) \Longleftrightarrow\left(\mathscr{R}^{*}\right) \text {. }
$$

If $u \in \mathscr{E}_{w}, u>0$, using also (2.8), it follows:

$$
\left(\mathscr{R}_{u}^{*}\right) \Longrightarrow\left(\mathscr{R}_{u}\right) \text {. }
$$

LEMma 2.1. Suppose that there exists an increasingly dense subset $D \subseteq \mathscr{E}_{w}$ such that for every $\alpha>0$ we have: $W_{\alpha}(D) \subseteq\left(\mathscr{E}_{w}\right)_{0}$ (resp. $W_{\alpha}(D) \subseteq$ $\left(\mathscr{E}_{w}\right)_{0}(u)$, for a fixed element $\left.u \in \mathscr{E}_{w}, u>0\right)$. Then $\mathscr{W}=\left(W_{\alpha}\right)_{a>0}$ satisfies $\left(\mathscr{R}^{*}\right)\left(\right.$ resp. $\left.\left(\mathscr{R}_{u}^{*}\right)\right)$.

Proof. Let us take $u \in \mathscr{E}_{w}, u>0, t \in\left(\mathscr{E}_{w}\right)_{0}$ and $\left(t_{n}\right)_{n \in N} \subseteq D$ with $t_{n} \nearrow t$. Then for any integer $k \geq 1$, there exists $n_{k} \in N$ such that $t \leq t_{n_{k}}+\frac{1}{k} u$. Hence $\left|W_{\alpha}(t)-W_{\alpha}\left(t_{n_{k}}\right)\right| \leq \frac{1}{k \alpha} u$, for any integer $k \geq 1$. Proposition 4.1.2 d) in [1] implies that $W_{a}(t)$ is $u$-continuous and the proof is finished.

Remark 2.2. Let $v \in \mathscr{E}_{w}, v>0$ be such that $W(v)$ is a nearly con- 
tinuous element of $\mathscr{E}_{\mathscr{}}$ (i.e. $W(v) \in \mathscr{E}_{\mathscr{W}}$ and there exists a sequence $\left(t_{n}\right)_{n \in N}$ $\subseteq\left(\mathscr{E}_{\mathscr{W}}\right)_{0}$ with $\left.W(v)=\sum_{n \in N} t_{n}\right)$. Then there exists $u \in \mathscr{E}_{\mathscr{W}}, u>0$ such that $\mathscr{W}=\left(W_{\alpha}\right)_{\alpha>0}$ satisfies condition $\left(\mathscr{R}_{u}^{*}\right)$.

Proof. From Proposition 5.6.1 in [1] there exists $u \in \mathscr{E}_{\mathscr{w}}, u>0$ such that $W(v)$ is $u$-continuous. Using (2.2), we obtain $W(t) \in\left(\mathscr{E}_{\mathscr{w}}\right)_{0}(u)$, for every $t \in\left(\mathscr{E}_{w}\right)_{0}$. Condition $\left(\mathscr{R}_{u}^{*}\right)$ now follows from the resolvent equation.

Remark 2.3. Let us suppose that $X$ is a locally compact space with countable base (with the associated Borel $\sigma$-field), $1 \in \mathscr{E}_{\mathscr{W}}$ and $W_{\alpha}(f)$ is continuous and tends to zero at infinity, for any $\alpha \geq 0$ and any bounded positive measurable function $f$ with compact support. Then $\mathscr{W}=\left(W_{\alpha}\right)_{\alpha>0}$ satisfies condition $\left(\mathscr{R}_{1}^{*}\right)$.

Proof. Let us first remark that every $t \in \mathscr{E}_{\mathscr{r}}$ is lower semi-continuous. We denote by $\mathscr{A}$ the set of all positive bounded Borel measurable functions with compact support. If $f \in \mathscr{A}$, using the complete maximum principle, it is easy to see that $W(f) \in\left(\mathscr{E}_{\mathscr{Y}}\right)_{0}$. The set $W(\mathscr{A})$ being increasingly dense in $\mathscr{E}_{\mathscr{r}}$, we obtain that the $\mathscr{W}$-excessive functions which are continuous and tend to zero at infinity are exactly the 1-continuous elements of $\mathscr{E}_{\mathscr{r}}$. We may now apply Lemma 2.1 , taking $D=W(\mathscr{A})$.

\section{§3. Integral representation of the ultrapotentials}

We maintain the context of the previous section.

For a convex subset $K$ of $\mathscr{E}_{r}$, let us denote by $\operatorname{ex}(K)$ the set of all extreme points of $K$.

Proposition 3.1. If $\alpha \geq 0$ and $u \in \mathscr{E}_{\mathscr{r}}, u>0$, then

$$
\operatorname{ex}\left(\tilde{\mathscr{U}}_{\alpha}(V) \cap K_{u}\right) \subseteq \bigcup_{0<\lambda \leq \infty} E(V, \lambda) .
$$

Proof. If $s \in \operatorname{ex}\left(\tilde{\mathscr{U}}_{\alpha}(V) \cap K_{u}\right) \backslash\{0\}$, then $\langle s, u\rangle=1$.

Let $\beta>0$ be fixed. We put

$$
a_{\beta}=\beta\left\langle V_{\alpha+\beta}(s), u\right\rangle \text {. }
$$

Thus $0 \leq a_{\beta} \leq 1$. If $a_{\beta}=0$, then $V_{\alpha+\beta}(s)=0, s=0$. If $a_{\beta}=1$, then $\left\langle s-\beta V_{\alpha+\beta}(s), u\right\rangle=0, \beta V_{\alpha+\beta}(s)=s, s \in E(V, \infty)$. Hence we may suppose that $0<a_{\beta}<1$.

If we put $s_{1}=\frac{1}{a_{\beta}} \beta V_{\alpha+\beta}(s)$ and $s_{2}=\frac{1}{1-a_{\beta}} \phi_{\beta}^{\alpha}(s)$, then it follows $s_{1}$, 
$s_{2} \in \tilde{\mathscr{U}}_{\alpha}(V) \cap K_{u}$. The equality $s=a_{\beta} s_{1}+\left(1-a_{\beta}\right) s_{2}$ and the extremality of $s$ imply that $s \in E_{\alpha+\beta}\left(V, \frac{a_{\beta}}{\beta}\right)$. (3.1) is now a consequence of (1.8).

Remark 3.2. a) Let $u \in \mathscr{E}_{\mathscr{W}}, u>0$ be such that $\mathscr{V}=\left(V_{\alpha}\right)_{\alpha>0}$ satisfies condition $\left(\mathscr{R}_{u}\right), \alpha \geq 0$ and $\beta, \lambda>0$. Then the restrictions of $V_{\beta}$ and $\phi_{\beta}^{\alpha}$ to $K_{u}$ are continuous. In addition, $E_{\alpha}(V, \lambda) \cap K_{u}, \tilde{\mathscr{U}}_{\alpha}(V) \cap K_{u}$ are compact convex subsets of $\mathscr{E}_{r}$.

b) If condition $(\mathscr{R})$ is satisfied, then $E_{\alpha}(V, \lambda)$, and $\tilde{\mathscr{U}}_{\alpha}(V)$ are closed convex subcones of $\mathscr{E}_{r}$, for any $\alpha \geq 0$ and $\lambda>0$.

Proof. Assertion a) is a direct consequence of condition $\left(\mathscr{R}_{u}\right)$ and (1.6). Assertion b) follows from a) and (2.10).

Let us point out that, even if condition $(\mathscr{R})$ is satisfied, the convex cone $\mathscr{U}(V)$ of all $V$-ultrapotentials is not necessarily a closed subset of $\mathscr{E}_{r}$ (see Remark 4.1).

If $M$ is a subset of $\mathscr{E}_{r}$, we denote by $\operatorname{cl} \operatorname{con}(M)$ the closed convex hull of $M$.

CoROLlaRy 3.3. a) Let $u \in \mathscr{E}_{\mathscr{w}}, u>0$ be such that condition $\left(\mathscr{R}_{u}\right)$ is satisfied. Then:

$$
\tilde{\mathscr{U}}(V) \cap K_{u}=\tilde{\mathscr{U}}_{\alpha}(V) \cap K_{u}, \quad \text { for any } \alpha>0 ;
$$

and

$$
\tilde{\mathscr{U}}(V) \cap K_{u}=\mathrm{cl} \cdot \operatorname{con}\left(\bigcup_{0<\lambda \leq \infty} E(V, \lambda) \cap K_{u}\right) .
$$

b) If condition ( $\mathscr{R})$ is satisfied, then

$$
\tilde{\mathscr{U}}(V)=\tilde{\mathscr{U}}_{\alpha}(V), \quad \text { for any } \alpha>0 \text {. }
$$

Proof. Let $\alpha>0$ be fixed. Then, by (1.3): $\tilde{\mathscr{U}}(V) \cap K_{u} \subseteq \tilde{\mathscr{U}}_{\alpha}(V) \cap K_{u}$. From (3.1) and (1.7) it follows:

$$
\operatorname{ex}\left(\tilde{\mathscr{U}}_{\alpha}(V) \cap K_{u}\right) \subseteq \tilde{\mathscr{U}}(V) \cap K_{u}
$$

and the equality (3.2), of compact convex sets (see Remark 3.2 a)), is now clear.

We have cl. $\operatorname{con}\left(\bigcup_{0<\lambda \leq \infty} E(V, \lambda) \cap K_{u}\right) \subseteq \tilde{\mathscr{U}}(V) \cap K_{u}$ and from (3.1) results (3.3),

Assertion b) follows from (3.2) and (2.9). 
Definition. If $h \in \bigcup_{0<\lambda<\infty} E(V, \lambda) \backslash\{0\}$, we define by $\Lambda(h)$ the uniquely defined positive real number such that

$$
V(h)=\Lambda(h) \cdot h
$$

and we put $\Lambda(h)=\infty$ if $h \in E(V, \infty)$.

Remark 3.4. If $u \in \mathscr{E}_{\mathscr{W}}, u>0$ is such that condition $\left(\mathscr{R}_{u}\right)$ is satisfied, then the above defined map $\left.\left.\Lambda: \bigcup_{0<\lambda \leq \infty} E(V, \lambda) \cap K_{u} \backslash\{0\} \rightarrow\right] 0, \infty\right]$ is continuous.

THEOREM 3.5. Let $\mathscr{V}=\left(V_{\alpha}\right)_{\alpha>0}$ and $\mathscr{W}=\left(W_{\alpha}\right)_{\alpha>0}$ be two submarkovian resolvents of kernels (with the initial kernels $V$ and $W$ proper) which are absolutely continuous and in duality with respect to a $\sigma$-finite measure. Suppose that $u \in \mathscr{E}_{\mathscr{w}}, u>0$ such that $\mathscr{V}=\left(V_{\alpha}\right)_{\alpha>0}$ satisfies condition $\left(\mathscr{R}_{u}\right)$.

If $s \in \mathscr{E}_{r}$ and $\langle s, u\rangle\langle\infty$, the following assertions are equivalent:

a) $s \in \mathscr{U}_{r}(V)$ (resp. $s \in \tilde{\mathscr{U}}(V)$ ).

b) There exist a finite positive Borel measure $\sigma$ on $] 0, \infty[$ (resp. on $] 0, \infty]$ ) and a family $\left(s_{\lambda}\right)_{0<\lambda<\infty}\left(\right.$ resp. $\left.\left(s_{\lambda}\right)_{0<\lambda \leq \infty}\right), s_{\lambda} \in E(V, \lambda)$ such that for every $t \in \mathscr{E}_{\mathscr{r}}$ the map $\lambda \mapsto\left\langle s_{\lambda}, t\right\rangle$ defined on ]0, $\infty[$ (resp. on ]0, $\infty]$ ) is $\sigma$-measurable and:

$$
\tilde{s}=\int \tilde{s}_{\lambda} d \sigma(\lambda)
$$

(i.e. $\langle s, t\rangle=\int\left\langle s_{\lambda}, t\right\rangle d \sigma(\lambda)$, for every $\left.t \in \mathscr{E}_{\mathscr{W}}\right)$.

Proof. We may suppose that $s \in K_{u}$.

"b) $\Rightarrow$ a)" Let $\alpha>0$ and $n \in N$ be fixed. If $t \in \mathscr{E}_{w}$, from (*) it follows:

$$
\langle s, t\rangle=\int\left(\frac{1+\alpha \lambda}{\lambda}\right)^{n}\left\langle V_{\alpha}^{n}\left(s_{\lambda}\right), t\right\rangle d \sigma(\lambda),
$$

where $\frac{1+\alpha \lambda}{\lambda}=\alpha$ if $\lambda=\infty$. Thus

$$
\int\left(\frac{1+\alpha \lambda}{\lambda}\right)^{n}\left\langle s_{\lambda}, W_{\alpha}^{n}(u)\right\rangle d \sigma(\lambda) \leq 1
$$

If we define on $\mathscr{E}_{\mathscr{W}}$ the map $\mu_{\alpha}^{n}$ by:

$$
\mu_{\alpha}^{n}(t)=\int\left(\frac{1+\alpha \lambda}{\lambda}\right)^{n}\left\langle s_{\lambda}, t\right\rangle d \sigma(\lambda), \quad t \in \mathscr{E}_{\mathscr{W}},
$$

then the above inequality implies that $\mu_{\alpha}^{n}$ is an $H$-integral on $\mathscr{E}_{\mathscr{w}}$. Moreover, with the identification given by (2.4), we have: 


$$
\mu_{\alpha}^{n}\left(\varepsilon_{x}\right)=\int\left(\frac{1+\alpha \lambda}{\lambda}\right)^{n} s_{\lambda}(x) d \sigma(\lambda)
$$

for any $x \in X$, where $\varepsilon_{x}$ is the $H$-integral on $\mathscr{E}_{\mathscr{r}}$ given by the Dirac measure at $x \in X$. Hence the function $s_{\alpha}^{n}: X \rightarrow \overline{\boldsymbol{R}}_{+}$defined by

$$
s_{\alpha}^{n}(x)=\int\left(\frac{1+\alpha \lambda}{\lambda}\right)^{n} s_{\lambda}(x) d \sigma(\lambda)
$$

is an element of $\mathscr{E}_{\mathscr{\gamma}}$ and $\tilde{s}_{\alpha}^{n}=\mu_{\alpha}^{n}$.

For every $t \in \mathscr{E}_{\mathscr{W}}$ we have $\left\langle V_{\alpha}^{n}\left(s_{\alpha}^{n}\right), t\right\rangle=\left\langle s_{\alpha}^{n}, W_{\alpha}^{n}(t)\right\rangle=\mu_{\alpha}^{n}\left(W_{\alpha}^{n}(t)\right)=\langle s, t\rangle$. It follows successively: $\overparen{V_{\alpha}^{n}\left(s_{\alpha}^{n}\right)}=\tilde{s}, V_{\alpha}^{n}\left(s_{\alpha}^{n}\right)=s, s \in \tilde{\mathscr{U}}_{\alpha}(V) \cap K_{u}=\tilde{\mathscr{U}}(V) \cap K_{u}$.

Let us remark that $s$ is purely excessive if the measure $\sigma$ is supported by $] 0, \infty[$ in the representation $(*)$.

Indeed, $\left\langle\alpha V_{\alpha}(s), t\right\rangle=\int \frac{\alpha \lambda}{1+\alpha \lambda}\left\langle s_{\lambda}, t\right\rangle d \sigma(\lambda)<\infty$, for any $t \in\left(\mathscr{E}_{w}\right)_{0}$. Letting $\alpha \searrow 0$ we obtain that

$$
\inf _{\alpha>0}\left\langle\alpha V_{\alpha}(s), t\right\rangle=0, \quad \text { for every } t \in\left(\mathscr{E}_{\mathscr{r}}\right)_{0},
$$

hence $s$ is purely excessive.

From Proposition $1.4 \mathrm{c}$ ) it now results that the proof of " $b$ ) $\Rightarrow a$ )" is complete.

"a) $\Rightarrow$ b)". If $s \in \tilde{\mathscr{U}}(V)$, then $s$ is an element of $\tilde{\mathscr{U}}(V) \cap K_{u}$, a metrisable compact convex subset of $\mathscr{E}_{V}$ (see Remark 3.2 a)). From the Choquet representation theorem there exists a finite measure $\varphi$ on $\operatorname{ex}\left(\tilde{\mathscr{U}}(V) \cap K_{u}\right) \backslash\{0\}$ with barycenter $s$. Then

$$
\tilde{s}=\int \tilde{h} d \varphi(h)
$$

(i.e. $\langle s, t\rangle=\int\langle h, t\rangle d \varphi(h)$, for any $t \in \mathscr{E}_{\mathscr{W}}$ ).

If $t \in\left(\mathscr{E}_{w}\right)_{0}$, we define the measure $\varphi_{t}$ on $\operatorname{ex}\left(\tilde{\mathscr{U}}(V) \cap K_{u}\right) \backslash\{0\}$ by:

$$
\varphi_{t}=\tilde{t} \cdot \varphi
$$

where $\tilde{t}$ is the $H$-integral on $\mathscr{E}_{\curlyvee}$ defined by $t$.

The measure $\varphi_{t}$ is finite and $\varphi_{t} \ll \varphi$ (i.e. $\varphi_{t}$ is absolutely continuous with respect to $\varphi$ ).

From (3.1) and Remark 3.4 it follows that we have a Borel measurable function $\left.\left.\Lambda: \operatorname{ex}\left(\tilde{\mathscr{U}}(V) \cap K_{u}\right) \backslash\{0\} \rightarrow\right] 0, \infty\right]$.

Let us denote by $\sigma$ and $\sigma_{t}$ the images of $\varphi$ and $\varphi_{t}$ under $\Lambda$. Hence 
$\boldsymbol{\sigma}$ and $\sigma_{t}$ are finite Borel measures on $\left.] 0, \infty\right]$ and $\sigma_{t} \ll \sigma$. More precisely

$$
\int g(\lambda) d \sigma_{t}(\lambda)=\int g(\Lambda(h))\langle h, t\rangle d \varphi(h), \quad g \in \mathscr{B}_{+},
$$

where $\mathscr{B}_{+}$denotes the set of all positive Borel measurable functions on $10, \infty]$.

In addition $\sigma_{t_{1}+t_{2}}=\sigma_{t_{1}}+\sigma_{t_{2}}$ if $t_{1}, t_{2} \in\left(\mathscr{E}_{\mathscr{r}}\right)_{0}$. It $t \in\left(\mathscr{E}_{\mathscr{r}}\right)_{0}$, by (2.2), there exists a positive real number $a_{t}$ such that $t \leq a_{t} \cdot u$, hence $\tilde{t} \leq a_{t}$ on $K_{u}$ and $\int g(\lambda) d \sigma_{t}(\lambda) \leq a_{t} \int g(\lambda) d \sigma(\lambda)$, for any $g \in \mathscr{B}_{+}$.

Let us denote by $f_{t}$ the Radon-Nikodim density of $\sigma_{t}$ with respect to $\boldsymbol{\sigma}$. Then $f_{t} \leq a_{t} \boldsymbol{\sigma}$-a.e. It follows $f_{t} \in \mathscr{L}^{\infty}(\boldsymbol{\sigma}), f_{t_{1}}+f_{t_{2}}=f_{t_{1}+t_{2}} \boldsymbol{\sigma}$-a.e., for any $t, t_{1}, t_{2} \in\left(\mathscr{E}_{w}\right)_{0}$ and $f_{t_{1}} \leq f_{t_{2}} \sigma$-a.e. if $t_{1} \leq t_{2}$. Using the lifting theorem of C. Ionescu-Tulcea, for every $t \in\left(\mathscr{E}_{\Downarrow}\right)_{0}$ we may choose $f_{t} \in \mathscr{L}^{\infty}(\sigma)$ such that $f_{t_{1}}+f_{t_{2}}=f_{t_{1}+t_{2}}$ on $\left.] 0, \infty\right]$ (with $\left.t_{1}, t_{2} \in\left(\mathscr{E}_{\mathscr{w}}\right)_{0}\right)$ and $f_{t_{1}} \leq f_{t_{2}}$ on $\left.] 0, \infty\right]$ if $t_{1} \leq t_{2}$. For any $\lambda \in] 0, \infty]$ we have obtained an additive and increasing map $t \mapsto f_{t}(\lambda)$ from $\left(\mathscr{E}_{*}\right)_{0}$ into $\boldsymbol{R}_{+}$. Hence, by (2.5), there exists $s_{\lambda}^{\prime} \in \mathscr{E}_{\curlyvee}$ such that $\left\langle s_{\lambda}^{\prime}, t\right\rangle=f_{t}(\lambda)$, and the map $\lambda \mapsto\left\langle s_{\lambda}^{\prime}, t\right\rangle$ is $\sigma$-measurable for any $t \in \mathscr{E}_{\psi}$.

If $t \in \mathscr{E}_{\mathscr{w}}$ and $g \in \mathscr{B}_{+}$, from (3.4) results:

$$
\int g(\lambda) \tilde{s}_{\lambda}^{\prime}(t) d \sigma(\lambda)=\int g(\Lambda(h))\langle h, t\rangle d \varphi(h) .
$$

Thus, for every $\alpha>0$ :

$$
\begin{aligned}
& \int g(\lambda)\left\langle s_{\lambda}^{\prime}, W_{\alpha}(t)\right\rangle d \sigma(\lambda)=\int g(\Lambda(h))\left\langle h, W_{\alpha}(t)\right\rangle d \varphi(h) \\
& =\int \frac{\Lambda(h)}{1+\alpha \Lambda(h)} g(\Lambda(h))\langle h, t\rangle d \varphi(h)=\int \frac{\lambda}{1+\alpha \lambda} g(\lambda)\left\langle s_{\lambda}^{\prime}, t\right\rangle d \sigma(\lambda) .
\end{aligned}
$$

It follows $\left\langle V_{\alpha}\left(s_{\lambda}^{\prime}\right), t\right\rangle=\frac{\lambda}{1+\alpha \lambda}\left\langle s_{\lambda}^{\prime}, t\right\rangle \sigma$-a.e. From (2.1) it derives that there exists a Borel measurable set $B$ of $] 0, \infty]$ such that $\sigma(B)=0$ and $s_{\lambda}^{\prime} \epsilon$ $E(V, \lambda)$ for every $\lambda \notin B$. Let us put:

$$
s_{\lambda}= \begin{cases}s_{\lambda}^{\prime}, & \text { if } \lambda \notin B \\ 0, & \text { if } \lambda \in B .\end{cases}
$$

Hence $s_{\lambda} \in E(V, \lambda)$, for every $\left.\left.\lambda \in\right] 0, \infty\right]$, the map $\lambda \mapsto\left\langle s_{\lambda}, t\right\rangle$ is $\sigma$-measurable for any $t \in \mathscr{E}_{\mathscr{V}}$ and $\langle s, t\rangle=\int\langle h, t\rangle d \varphi(h)=\int\left\langle s_{\lambda}, t\right\rangle d \sigma(\lambda)$.

Corollary 3.6. If, in Theorem 3.5, condition $(\mathscr{R})$ is satisfied, then we have a) $\Leftrightarrow$ b) for every $s \in \mathscr{E}_{r}$. 
Remark 3.7. If $s \in \tilde{\mathscr{U}}(V)$ has the representation (*) from the above theorem, then:

$$
s(x)=\int s_{\lambda}(x) d \sigma(\lambda), \quad \text { for any } x \in X
$$

In addition:

(3.6) the invariant part of $s$ is $\sigma(\{\infty\}) \cdot s_{\infty}$ and $\int_{] 0, \infty[} s_{\lambda} d \sigma(\lambda)$ is the purely excessive component of $s$.

Proof. (3.5) is a consequence of $\left(^{*}\right)$ since $\varepsilon_{x}$ is an $H$-integral on $\mathscr{E}_{r}$, for every $x \in X$. Assertion (3.6) is clear (see the final remark in the proof of "b) $\Rightarrow a)$ )").

We have the following unicity result:

Proposition 3.8. With the same assumptions as in Theorem 3.5, let $s \in \tilde{\mathscr{U}}(V)$ be such that $\langle s, u\rangle=1$. Then there exist a positive Borel measure $\sigma$ on $] 0, \infty]$ and a family $\left(s_{\lambda}\right)_{0<\lambda \leq \infty}, s_{\lambda} \in E(V, \lambda)$ such that for any $t \in \mathscr{E}_{\mathscr{W}}$ the map $\lambda \mapsto\left\langle s_{\lambda}, t\right\rangle$ is $\sigma$-measurable, $\left\langle s_{\lambda}, u\right\rangle=1$-a.e. and $s$ has the representation $(*)$. (In particular $\int d \boldsymbol{\sigma}=1$.) Moreover, $\boldsymbol{\sigma}$ and $\left(s_{\lambda}\right)_{0<\lambda \leq \infty}$ are uniquely determined. (Two $\sigma$-measurable maps $\lambda \mapsto s_{\lambda}$ and $\lambda \mapsto s_{\lambda}^{\prime}$ are equal if $s_{\lambda}=s_{\lambda}^{\prime}$ $\sigma$-a.e.)

Proof. By Theorem 3.5 there exists a finite Borel measure $\boldsymbol{\sigma}^{\prime}$ on ]0, $\infty$ ] and a family $\left(s_{\lambda}^{\prime}\right)_{0<\lambda \leq \infty}, s_{\lambda}^{\prime} \in E(V, \lambda)$ such that the map $\lambda \mapsto\left\langle s_{\lambda}^{\prime}, t\right\rangle$ is $\boldsymbol{\sigma}^{\prime}$-measurable for any $t \in \mathscr{E}_{\mathscr{r}}$ and $\tilde{s}=\int \tilde{s}_{\lambda}^{\prime} d \boldsymbol{\sigma}^{\prime}(\lambda)$. Since $\int\left\langle s_{\lambda}^{\prime}, u\right\rangle d \boldsymbol{\sigma}^{\prime}(\lambda)<\infty$, we may suppose that $\left\langle s_{\lambda}^{\prime}, u\right\rangle<\infty$ for every $\left.\left.\lambda \in\right] 0, \infty\right]$. Let us put

$$
s_{\lambda}=\left\{\begin{array}{cl}
\frac{1}{\left\langle s_{\lambda}^{\prime}, u\right\rangle}, & \text { if } s_{\lambda}^{\prime} \neq 0 \\
0, & \text { if } s_{\lambda}^{\prime}=0 .
\end{array}\right.
$$

and

$$
d \sigma(\lambda)=\left\langle s_{\lambda}^{\prime}, u\right\rangle d \sigma^{\prime}(\lambda) .
$$

Then $\sigma$ and $\left(s_{\lambda}\right)_{0<\lambda \leq \infty}$ satisfy the required conditions.

In order to discuss the unicity, let us point out the following simple consequence of Stone-Weierstrass theorem:

(3.7) If $\sigma, \sigma^{\prime \prime}$ are positive finite Borel measures on $] 0, \infty[$ such that

$$
\int\left(\frac{\lambda}{1+\lambda}\right)^{n} d \sigma(\lambda)=\int\left(\frac{\lambda}{1+\lambda}\right)^{n} d \sigma^{\prime \prime}(\lambda), \quad n \geq 0,
$$

then $\sigma=\sigma^{\prime \prime}$. 
Let $\sigma^{\prime \prime},\left(s_{\lambda}^{\prime \prime}\right)_{0<\lambda \leq \infty}$ be another system which satisfies the required conditions. From (3.6) we may suppose that $\sigma$ and $\sigma^{\prime \prime}$ are carried by ]0, $\infty$ [. Then $1=\langle s, u\rangle=\int d \sigma=\int d \sigma^{\prime \prime}$ and for any integer $n \geq 1$ we have $\overparen{V_{1}^{n}(s)}$

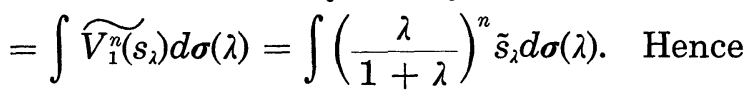

$$
\int\left(\frac{\lambda}{1+\lambda}\right)^{n}\left\langle s_{\lambda}, t\right\rangle d \sigma(\lambda)=\int\left(\frac{\lambda}{1+\lambda}\right)^{n}\left\langle s_{\lambda}^{\prime \prime}, t\right\rangle d \sigma^{\prime \prime}(\lambda),
$$

for any $t \in \mathscr{E}_{\mathscr{r}}$ and $n \geq 0$.

Applying (3.8) for $t=u$, from (3.7) we obtain: $\sigma=\sigma^{\prime \prime}$.

If $t \in\left(\mathscr{E}_{*}\right)_{0}$, let $a_{t}$ be $a$ positive real number such that $t \leq a_{t} u$, hence $\left\langle s_{\lambda}, t\right\rangle \leq a_{t}$ for any $\lambda>0$. Thus $\left\langle s_{\lambda}, t\right\rangle \cdot d \sigma(\lambda)$ and $\left\langle s_{\lambda}^{\prime \prime}, t\right\rangle \cdot d \sigma(\lambda)$ are finite measures on $] 0, \infty\left[\right.$. Again (3.8) and (3.7) imply that $\left\langle s_{\lambda}, t\right\rangle \cdot d \sigma(\lambda)=$ $\left\langle s_{\lambda}^{\prime \prime}, t\right\rangle \cdot d \sigma(\lambda)$. Hence, for any $t \in\left(\mathscr{E}_{\mathscr{r}}\right)_{0},\left\langle s_{\lambda}, t\right\rangle=\left\langle s_{\lambda}^{\prime \prime}, t\right\rangle \sigma-a . e$, and from (2.1) it follows: $s_{\lambda}=s_{\lambda}^{\prime \prime} \sigma$-a.e.

We finish this section underlining some additional connections with the duality for standard $H$-cones. As a consequence, our hypothesis should be slightly modified.

The dual of the standard $H$-cone $\mathscr{E}_{\gamma}$ is by definition the set of all $H$-integrals on $\mathscr{E}_{\gamma}$ and will be denoted by $\mathscr{E}_{\gamma}^{*}$.

We have already observed, see (2.4), that $\mathscr{E}_{r}^{*}=\mathscr{E}_{\psi}$.

The equality (2.6) shows that, considered as a map on $\mathscr{E}_{r}^{*}, W_{\alpha}$ is exactly the dual of the $H$-morphism $V_{\alpha}$, for any $\alpha>0$. (With the notations of [1]: $\mathrm{V}_{\alpha} \in \operatorname{Hom}\left(\mathscr{E}_{r}, \mathscr{E}_{r}\right)$ and $\left.W_{\alpha}=V_{\alpha}^{*}.\right)$

The strictly positive $\mathscr{W}$-excessive functions $u \in \mathscr{E}_{\mathscr{W}}, u>0$ are precisely the weak units of the standard $H$-cone $\mathscr{E}_{r}^{*}$.

Summarizing, we have the following:

Remark 3.9. The results of this section are valid for the following set up:

$\mathscr{V}$ is a submarkovian resolvent of kernels (with the initial kernel proper) which is absolutely continuous with respect to a $\sigma$-finite measure, and it is such that $\mathscr{E}_{\curlyvee}$ coincides with its bidual.

\section{§4. Examples}

ExAmple 1 (The Bernstein theorem). Let $(X, \mathscr{B})$ be the measurable space ]0, $\infty$ [ (endowed with the $\sigma$-algebra of Borel sets), and let the sub- 
markovian resolvents of kernels $\mathscr{V}=\left(V_{\alpha}\right)_{\alpha>0}$ and $\mathscr{W}=\left(W_{\alpha}\right)_{\alpha>0}$ on $X$ be defined by:

$$
\begin{aligned}
& V_{\alpha}(f)(x)=e^{\alpha x} \int_{x}^{\infty} e^{-\alpha u} f(u) d u, \\
& W_{\alpha}(f)(x)=e^{-\alpha x} \int_{\infty}^{x} e^{\alpha u} f(u) d u, \quad \text { for any } f \in \mathscr{F} \text { and } x \in X .
\end{aligned}
$$

Thus $\mathscr{V}$ and $\mathscr{W}$ are absolutely continuous and in duality with respect to the Lebesgue measure. We may verify that $s \in \mathscr{E}_{*}$ is universally continuous iff it is continuous, bounded and there exists $x_{0}>0$ such that $s\left(x_{0}\right)=0$. As a consequence, condition $\left(\mathscr{R}^{*}\right)$ is satisfied. Also, it is easy to see that:

a) The positive constant functions are the only invariant excessive functions. If $0<\lambda<\infty$ then $E(V, \lambda)=\left\{x \mapsto c \cdot e^{-(1 / \lambda) x} / c \in \boldsymbol{R}_{+}\right\}$.

b) A real function $s$ is a $V$-ultrapotential iff $s$ is complete monotone (i.e. $s$ is infinitly differentiable and $(-1)^{n} D^{n} s \geq 0$, for any integer $n \geq 0$, where $D^{n} s$ is the $n$-th derivative of the function $s$ ) and $\lim _{x \rightarrow \infty} s(x)=0$.

c) $\tilde{\mathscr{U}}(V)$ coincides with the convex cone of all complete monotone functions.

Let us point out that the intermediate characterization for the complete monotone functions established in the Choquet's proof of Bernstein theorem (see [4] or T. 40 in [8]) may be considered as the analogous, with the associated semigroup of kernels language, of that given by the above assertion c) in the resolvent terms.

From Corollary 3.6 we obtain the Bernstein theorem.

Remark 4.1. The convex cone $\mathscr{U}(V)$ is not closed.

Proof. The constant function 1 (which is not a $V$-ultrapotential) is the limit of the following sequence $\left(s_{n}\right)_{n \in N} \subseteq \mathscr{U}(V): s_{n}(x)=e^{-(1 / n) x}, x \in X$.

ExAmple 2 (The elliptic case). With the notations of Section 2, if $\mathscr{E}$ is a convex subcone of $\mathscr{E}_{r}$ and $u \in \mathscr{E}_{r}, u>0$, then the convex cone $\mathscr{E}_{u}$ is defined by:

$$
\mathscr{E}_{u}=\{s \in \mathscr{E} \mid\langle s, u\rangle\langle\infty\} .
$$

Proposition 4.2. Let $V$ be such that it induces a bounded linear operator on $L^{1}(\mathfrak{m})$. Suppose that $1 \in \mathscr{E}_{\mathscr{W}}$, condition $\left(\mathscr{R}_{1}\right)$ and also the following assumptions are satisfied: 
(C) Some power of $V$ is compact;

(E) $V(f)$ is strictly positive if $f \in \mathscr{F}$ and $V(f) \neq 0$.

Then, the convex cone $\mathscr{U}(V)_{1}$ is one dimensional (i.e. there exists $s \in \mathscr{U}(V)_{1}$, $s \neq 0$, and any other element of $\mathscr{U}(V)_{1}$ is proportional with $\left.s\right)$.

Proof. Condition (E) implies that $V$ is an irreducible kernel operator on $L^{1}(\mathfrak{m})$ in the sense of H.H. Schaefer, and from Theorem 6.6, ch. $\mathrm{V}$ in [10] it follows that there exists exactly one eigenvalue $r>0$ for $V$ which has positive eigenvectors and the corresponding eigenspace is one dimensional. Remarking that every element of $E(V, \lambda)_{1}$ is $\mathrm{m}$-integrable for $0<\lambda<\infty$, we conclude that $E(V, r)_{1}$ is one dimensional and $E_{i}^{\prime}(V, \lambda)=\{0\}$ if $\lambda \neq r, \lambda \in R$. Theorem 3.5 implies now that $\mathscr{U}(V)_{1}=E(V, r)_{1}$ and the proof is finished.

Remark 4.3. a) If in the above proposition we know in addition that every $V$-ultrapotential is m-integrable, then $\mathscr{U}(V)=\mathscr{U}(V)_{1}$, hence $\mathscr{U}(V)$ is one dimensional.

b) Condition (E) is equivalent with the following one:

any $\mathscr{V}$-excessive function is strictly positive.

Thus, in connection with the characterization of the elliptic harmonic spaces (see [5]), condition (E) might be understood as an "ellipticity property" of the kernel $V$.

CoRollary 4.4. Let $V$ be the Green kernel of a second order elliptic differential operator (with indefinite differentiable coefficients) in a bounded domain (with a sufficiently regulate boundary) of $\boldsymbol{R}^{n}$. Then $\mathscr{U}(V)$ is one dimensional.

Proof. In this case $V$ and $W$ are bounded kernels. Hence $V$ is a bounded linear operator on $L^{1}(\mathfrak{m})$. Also, condition (E) is fulfilled.

The structure of the associated Green function (see [2] page 275) implies that there exists an integer $k>0$ such that $V^{k}$ maps $L^{1}(\mathfrak{m})$ in $L^{\infty}(\mathfrak{m})$. By Example 5, page 337 in [10], it results that condition (C) is satisfied. From Remark 2.3 it is easy to see that condition $\left(\mathscr{R}_{1}^{*}\right)$ is also satisfied.

The desired assertion is obtained from the above proposition. (See also Remark 4.3 a).)

Let us point out that Corollary 4.4 shows that Proposition 4.2 is a generalization of the results of this type obtained in [2] Theorem 12 and 
[7] Corollary 83.

Indeed, we have already observed (see Remark 1.1) that the "ultrapotentials associated to a second order elliptic differential operator" in [2] are from $\mathscr{U}(V)$.

By Proposition 81 and the equality (4.7) in [7] we obtain that in this case the "completely $L$-superharmonic functions with zero conditions" are also elements of $\mathscr{U}(V)$.

\section{REFERENCES}

[1] N. Boboc, Gh. Bucur and A. Cornea, Order and convexity in potential theory: $H$-cones, Lecture Notes in Math., 853, Springer, Berlin-Heidelberg-New York, 1981.

[2] N. Boboc and P. Mustaţã, Fonctions complètement surharmoniques associées aux operateurs differentiels de second ordre du type elliptique, Ann. Fac. Sci. de Kinshasa, Zaire; Section Math-Phys., 1 (1975), 249-281.

[ 3 ] N. Boboc and M. M. Nkomba-Tshola, Eléments complètement excessifs par rapport à une résolvante, Ann. Fac. Sci. de Kinshasa, Zair; Section Math.-Phys., 2 (1976), $1-30$.

[4] G. Choquet, Deux exemples classiques de représentation intégral, Enseignement Math., 15 (1969), 63-75.

[5] C. Constantinescu and A. Cornea, Potential theory on harmonic spaces, Springer, Berlin-Heidelberg-New York, 1972.

[6] M. Itô, Positive eigen elements for an infinitesimal generator of a diffusion semigroup and their integral representations, In: Potential Theory Copenhagen 1979, Lecture Notes in Math., 787, Springer, 1980.

[7] M. Itô and N. Suzuki, Completely superharmonic measures for the infinitesimal generator $A$ of a diffusion semi-group and positive eigen elements of $A$, Nagoya Math. J., 83 (1981), 53-106.

[ 8 ] P.-A. Meyer, Probability and Potentials, Blaisdell Publishing Comp. 1966.

[9] - Processus de Markov: la frontière de Martin, Lecture Notes in Math., 77, Springer, Berlin-Heidelberg-New York, 1968.

[10] H. H. Schaefer, Banach lattices and positive operators, Springer, Berlin-Heidelberg-New York, 1974.

Department of Mathematics, INCREST,

Bd. Pacii 220, 79622 Bucharest, Romania 